\title{
IDENTIFICATION OF COORDINATION MOTOR ABILITIES DETERMINING THE SPORTS SKILL LEVEL IN ELITE MALE BADMINTON PLAYERS
}

original paper

( ) University School of Physical Education in Wroclaw

DOI: https://doi.org/10.5114/hm.2021.98459

\author{
JANUSZ JAWORSKI ${ }^{1}$, GRZEGORZ LECH ${ }^{1}$, TADEUSZ AMBROŻY ${ }^{1}$, MICHAŁ $\dot{Z} A K^{2}$ \\ ${ }^{1}$ University of Physical Education in Krakow, Faculty of Physical Education and Sport, Institute of Sport Sciences, \\ Department of Sport Theory and Kinesiology, Krakow, Poland \\ ${ }^{2}$ University of Physical Education in Krakow, Faculty of Physical Education and Sport, Institute of Sport Sciences, \\ Department of Sports and Recreational Games, Krakow, Poland
}

\section{ABSTRACT}

Purpose. The main aim of this study was to evaluate the effect of coordination motor abilities on the sports skill level in elite male badminton players.

Methods. The study group consisted of 12 male players from the Polish national badminton team. The scope of the research included measurement of basic anthropometric features and selected coordination motor abilities. Using the Spearman's rank correlation coefficient, statistically significant cause and effect relations were identified between the sports skill level and the analysed coordination abilities. Multiple determination coefficients were computed to analyse the impact of individual coordination abilities on the sports skill level.

Results. The multiple determination coefficients showed that the sports skill level of the badminton players was most strongly determined by spatial orientation (ca. $42 \%$ ), followed by frequency of movements (28\%) and kinaesthetic differentiation (ca. 17\%). Conclusions. It seems that these coordination abilities should be taken into careful consideration in technical and tactical training of badminton players since they largely determine their level of achievement.

Key words: badminton, elite, coordination motor abilities, visual information, ranking

\section{Introduction}

The origins of badminton as a game date back to ancient times; nowadays, it is estimated that more than 220 million people around the world are involved in this sport recreationally or professionally [1]. In some regions of the world, especially in Asian countries, its popularity is even higher than that of football $[2,3]$.

It is critical for the effectiveness of athletic training to comprehensively identify the determinants of high sports skill level of players [4]. Badminton is dominated by open movement structures which are performed within time limitations. During a game, players perform various types of movements, such as sudden starts and stops, high intensity movements, accelerations, running with directional changes on the court, quick shuttlecock strokes, jumps, etc. [5-7]. The complex character of the game requires perfect performance of highly complex movement tasks and adaptation to frequent changes in the situation on the court [1]. Furthermore, a badminton player has to process a lot of information; this involves making rapid decisions whose accuracy determines the success of the movement sequence applied and affects the effectiveness of playing and the final score [8]. However, the identification, selection, and accurate characterization of the determinants of winning are substantially complex. Badminton is played using additional equipment (extending the hand with a racket), which makes it even more complicated to perform effective moves. It can therefore be assumed that a high level of coordination abilities should play a very important role in achieving champion-level performance. This is suggested by the complex nature of the game, which requires the use of perfect movement activities of high complexity and adaptation to constantly changing situations on the court.

Correspondence address: Janusz Jaworski, University of Physical Education in Krakow, Faculty of Physical Education and Sport, Institute of Sport Sciences, Department of Sport Theory and Kinesiology, al. Jana Pawła II 78, 31-571 Krakow, Poland, e-mail: janusz.jaworski@awf.krakow.pl

Received: May 17, 2019

Accepted for publication: January 8, 2020

Citation: Jaworski J, Lech G, Ambroży T, Żak M. Identification of coordination motor abilities determining the sports skill level in elite male badminton players. Hum Mov. 2021;22(1):9-15; doi: https://doi.org/10.5114/hm.2021.98459. 
Analysis of badminton matches shows that speed and endurance are the key to success. Anaerobic exercise is performed during single actions which usually take 3-10 seconds. Further, aerobic exercise is related to the total duration of the match, which usually lasts 40-60 minutes [9-13]. During a match, around 60-70\% of energy is supplied from aerobic sources, whereas the remaining $30-40 \%$ is generated by anaerobic sources [1].

Scientific studies concerning badminton have mostly referred to isolated motor skills, but the mechanisms of the physiological aspects of athletes' preparation have also been well documented (e.g. [1, 14-18]). The determinants of the level of somatic development of badminton players have been thoroughly explored, too. A relatively small variation in somatic characteristics has been observed in badminton players from such countries as Nigeria, Malaysia, Poland, Indonesia, Turkey, Spain, Denmark, the Czech Republic, South Africa, and Germany [1]. According to the classification of somatotypes proposed by Sheldon, badminton players are characterized by high indices of mesomorphy and ectomorphy [1]. In the studies analysed, mean values were 3.6 for mesomorphy and 3.1 for ectomorphy.

Compared with the studies of physiological and somatic aspects, explorations of the importance of coordination motor abilities in badminton are rare. Therefore, there is a need to pay more attention to phenomena relating to the identification and prioritization of coordination abilities and their importance in the achievement of high sports performance.

The main aim of this study was to evaluate the effect of coordination motor abilities on the sports skill level of male players from the Polish national badminton team. The following research questions were asked in the study:

- Which neurofunctional abilities are of key importance to the sports skill level in badminton players?

- What is the order of importance of the coordination variables that determine success in badminton?

\section{Material and methods}

\section{Participants}

The study group consisted of 12 male players from the Polish national badminton team. All tests were conducted in November 2017 during the competitive season. Badminton players were enrolled in a special training program conducted during a year-long training camp. During their stay at a sports centre, the players participated in training divided into 3 sessions a day, 5 times a week. The training sessions included improvement of technique, motor skills, and playing skills during preparation matches. Furthermore, the study group was subjected to regular physiotherapeutic procedures. Other days of the week were spent on participation in national and international badminton tournaments.

The sports skill level of the tested players was determined on the basis of classification lists drawn up by the Polish Badminton Association at the end of the season [19]. The observed population was a group of players competing in top-rank national and international tournaments. Analysis of the level of achievement of these badminton players reveals that all of them had won the highest trophies in national competitions, whereas some of them had participated in the Olympic Games and world championships; therefore, the competitors were elite Polish badminton players.

The training experience of the participants was verified in interviews with each of them; its mean duration was 12.75 years $\pm 3.13 S D$.

\section{Scope of the study}

Somatic characteristics were measured by using the Martin's technique. These included the following variables: body height $(b-v)$, body mass, lean body mass, and fat mass, evaluated with the Tanita TBF-551 body composition analyser. Table 1 presents the basic statistical characteristics of age and selected somatic parameters of the badminton players studied.

Table 1. Statistical characteristics of basic somatic parameters and age of the study participants

\begin{tabular}{lrcr}
\hline Variable & \multicolumn{1}{c}{} & $S D$ & $V[\%]$ \\
\hline Body height $[\mathrm{cm}]$ & 179.82 & 6.87 & 3.82 \\
Body mass $[\mathrm{kg}]$ & 75.55 & 7.62 & 10.08 \\
Body mass index $\left[\mathrm{kg} / \mathrm{m}^{2}\right]$ & 23.69 & 2.08 & 8.78 \\
Gyko height $[\mathrm{cm}]$ & 148.13 & 7.21 & 4.86 \\
Chronological age [years] & 21.67 & 0.88 & 6.90 \\
\hline
\end{tabular}

Selected coordination abilities related to contemporary classifications of human motor activity were also examined in the study [20]. For this purpose, a set of Jaworski's computer tests [21] was used to evaluate the following:

- simple reaction time to visual stimuli (10 stimuli), score in milliseconds;

- simple reaction time to auditory stimuli (10 stimuli), score in milliseconds; 
- selective reaction time to auditory and visual stimuli (20 stimuli), score in milliseconds;

- kinaesthetic differentiation of temporal movement parameters, score in pixels;

- frequency of movements, score in the number of touched squares;

- coupled motions, score in seconds;

- visual-motor coordination (eye-hand coordination), score in seconds;

- spatial orientation, score in seconds.

The coordination motor abilities were measured and the adopted procedure can be briefly described as follows [21]:

- reaction time to visual or auditory stimuli: after a stimulus was presented, the participants were supposed to click the left button of the mouse as quickly as possible;

- selective reaction time: depending on the stimulus (auditory or visual), the participant clicked the respective button of the mouse as quickly as possible;

- kinaesthetic differentiation: the participants predicted the time necessary to fill in a rectangle; the result of the test was the mean absolute deviation from the pattern, expressed in pixels;

- frequency of movements: within a timeframe of $15 \mathrm{~s}$, the participants touched 2 squares alternately with the tablet's stylus;

- coupled motions: the participants were to drag a square with the tablet's stylus through a special maze as quickly as possible and with lowest possible number of mistakes;

- eye-hand coordination: the participants used the tablet's stylus to touch a square which was flashed on the screen as quickly as possible;

- spatial orientation: the participants were shown a coordinate plane with 2 red squares along the $x$ and $y$ axes; the task was to use the tablet's stylus to plot as quickly as possible the point on the grid where the 2 red squares would intersect.

\section{Statistical analysis}

The following methods of statistical analysis were used to provide the answers to the research questions:

1. The Shapiro-Wilk test served to examine all variables for normal distribution and Levene's test was employed to assess the homogeneity of variances.

2. Basic descriptive statistics of the tested variables (arithmetic mean, standard deviation, coefficient of variation) were calculated.

3 . The correlation matrix between the tested coordination abilities was computed. Next, the Spearman's rank correlation coefficients were calculated between the sports skill level and selected coordination abilities. This allowed the coordination abilities to be chosen for further analysis which were most strongly correlated with the response (dependent) variable and at the same time not correlated with each other [22].

4. Multiple determination coefficients were computed to analyse the impact of individual coordination abilities on the sports skill level. The procedure of forward selection was adopted for the choice of input variables for the model. A variable was input into the model only when it was possible to reject the zero hypothesis $(F$-Snedecor statistic, $p<0.05)$. The threshold for inputting the variable into the multiple regression model was adopted as $F \geq 3$.

\section{Ethical approval}

The research related to human use has complied with all the relevant national regulations and institutional policies, has followed the tenets of the Declaration of Helsinki, and has been approved by the Bioethics Committee at the Regional Medical Chamber in Krakow, Poland (approval No.: 159/KBL/OIL/2017).

\section{Informed consent}

Informed consent has been obtained from all individuals included in this study.

\section{Results}

Correlation matrices between the tested coordination abilities are presented in Table 2; all correlation coefficients turned out to be statistically insignificant and ranged from -0.46 to 0.54 . The strongest correlations were observed between spatial orientation and visual-motor coordination, and between spatial orientation and coupled motions. In all cases, no statistically significant correlations were found, which allows the variables to be treated as unrelated.

Table 3 presents Spearman's rank correlation coefficients between sports skill level and selected coordination abilities. Only in the case of one variable (spatial orientation) was the coefficient statistically significant. Relatively high correlation coefficients were also obtained between sports skill level and frequency of movements, reaction time to visual stimuli, and eyehand coordination.

The variables which were finally input into the regression model were selected on the basis of the values of Spearman's rank correlation coefficients between the sports skill level and the analysed coordination variables. In accordance with the adopted methodology, 


\section{HUMAN MOVEMENT}

J. Jaworski, G. Lech, T. Ambroży, M. Żak, Identification of coordination abilities

Table 2. Correlation matrices between tested coordination motor abilities

\begin{tabular}{|c|c|c|c|c|c|c|c|c|}
\hline \multirow{2}{*}{ Variable } & \multicolumn{8}{|c|}{ Variable } \\
\hline & $\mathrm{KD}$ & $\mathrm{FM}$ & RTv & RTa & RTs & $\mathrm{CM}$ & EHc & SP \\
\hline KD & 1.00 & 0.18 & -0.35 & 0.17 & -0.46 & 0.16 & -0.32 & -0.24 \\
\hline $\mathrm{FM}$ & & 1.00 & 0.10 & 0.07 & -0.45 & 0.15 & 0.37 & 0.29 \\
\hline RTV & & & 1.00 & 0.51 & 0.32 & -0.14 & -0.11 & -0.04 \\
\hline RTa & & & & 1.00 & -0.07 & -0.11 & 0.08 & 0.21 \\
\hline RTs & & & & & 1.00 & -0.14 & 0.04 & 0.09 \\
\hline $\mathrm{CM}$ & & & & & & 1.00 & 0.41 & 0.54 \\
\hline EHc & & & & & & & 1.00 & 0.54 \\
\hline SP & & & & & & & & 1.00 \\
\hline
\end{tabular}

KD - kinaesthetic differentiation of temporal movement parameters, FM - frequency of movements, RTV - reaction time to visual stimuli, RTa - reaction time to auditory stimuli, RTs - selective reaction time (to auditory or visual stimuli), $\mathrm{CM}$ - coupled motions, EHc - eye-hand coordination, SP - spatial orientation

Table 3. Basic statistical characteristics of the tested variables and Spearman's rank correlations between sports skill level and the variables tested

\begin{tabular}{lcrrr}
\hline Variable & Sports skill level & $\bar{x}$ & $S D$ & $V[\%]$ \\
\hline Kinaesthetic differentiation [pixel] & 0.28 & 28.75 & 12.53 & 43.58 \\
Frequency of movements [n] & -0.56 & 50.33 & 6.92 & 13.74 \\
Reaction time to visual stimuli [ms] & -0.43 & 220.83 & 12.56 & 5.68 \\
Reaction time to auditory stimuli [ms] & 0.04 & 195.75 & 13.78 & 7.03 \\
Selective reaction time [ms] & 0.03 & 319.92 & 33.53 & 10.48 \\
Coupled motions [s] & -0.36 & 40.58 & 5.66 & 13.94 \\
Eye-hand coordination [s] & 0.48 & 34.83 & 3.41 & 9.79 \\
Spatial orientation [s] & $-0.63^{*}$ & 48.75 & 4.69 & 9.62 \\
\hline
\end{tabular}

${ }^{*} p<0.05$

Table 4. Multiple regression results for the sports skill level depending on selected coordination motor abilities

\begin{tabular}{lcrrr} 
& $\begin{array}{c}\text { Unstandardized } \\
\text { coefficients }\end{array}$ & Standard error & $t$-test & $p$ \\
\hline Absolute term & 128.0599 & 24.20294 & 5.29109 & $0.001^{*}$ \\
Spatial orientation & -1.2365 & 0.47988 & -2.57664 & $0.032^{*}$ \\
Kinaesthetic differentiation & 0.6348 & 0.14090 & 4.50559 & $0.001^{*}$ \\
Frequency of movements & -1.4013 & 0.32163 & -4.35691 & $0.002^{*}$ \\
\hline
\end{tabular}

${ }^{*} p<0.05$

Table 5. Multiple determination coefficients between the sports skill level and the tested coordination abilities

\begin{tabular}{lcccccc}
\hline Variable input into the model & Step & $R$ & $R^{2}$ & $R^{2}$, change & $F$ & 7.366 \\
\hline Spatial orientation & 1 & 0.651 & 0.424 & 0.424 & $0.021^{*}$ \\
Kinaesthetic differentiation & 2 & 0.775 & 0.601 & 0.176 & 3.993 & 0.076 \\
Frequency of movements & 3 & 0.939 & 0.881 & 0.280 & 18.982 & $0.002^{*}$ \\
\hline
\end{tabular}

${ }^{*} p<0.05$ 
the variables most correlated with the sports skill level and at the same time not correlated with each other were selected (Tables 2 and 3). Consequently, 6 variables were finally input into the model, while selective reaction time and reaction time to auditory stimuli were rejected. Multiple regression results are shown in Table 4.

On the basis of the data presented in Table 4, the regression functions calculated with the least-squares method were obtained as follows:

$$
\begin{aligned}
\mathrm{y}=-1.2365 \mathrm{SP}+ & 0.6348 \mathrm{KD}+-1.4013 \mathrm{FM}+ \\
& +128.0599
\end{aligned}
$$

where SP - spatial orientation, KD - kinaesthetic differentiation, FM - frequency of movements.

For the equation, the following values were obtained: $R^{2}=0.88 ; R^{2}$ corrected $=0.83 ; F(3,8)=19.884$.

In the context of the assumed aims of the study, the analysis of multiple determination coefficients is most interesting as it allows evaluation of the influence of individual coordination abilities on the sports skill level of badminton players. This information is presented in Table 5.

Sports skill level is most strongly determined by spatial orientation (ca. 42.4\%); the next variable qualified for the model is kinaesthetic differentiation of movements (ca. 17.7\%); in total, both variables explain ca. $60 \%$. It should be noted that for the variable of kinaesthetic differentiation, the value of the statistic $F$ is greater than the adopted criterion $(F \geq 3)$, but the variable was input into the model with $p$ slightly below the set level of significance. Inputting the next variable to the model, i.e. frequency of movements, explains other $28 \%$ of variability. The whole system of variables representing neurofunctional abilities explains $88 \%$ of the sports skill level of players.

\section{Discussion}

As was emphasized in the Introduction section, the problem of the effect of neurophysiological abilities on the level of achievement in the training of badminton players has been little explored to date. Badminton is one of the fastest racket sports, with maximum shuttlecock flight speed reaching over $400 \mathrm{~km} / \mathrm{h}$ [1, 23, 24]. Therefore, players are challenged with high requirements in the following areas: flexibility of nervous processes, divisibility of and alternating attention, precision of performance of activities in time and space, making appropriate decisions in response to competitive pressure, anticipating the opponent's actions, and performing effective counteractions [25, 26]. Therefore, top athletes must be characterized not only by a high level of endurance and appropriate motor skills, but also by a high level of specific coordination motor abilities, which can be understood in this case to mean somehow natural aptitudes (of neurophysiological nature). During a match, players have to process a lot of information and have very little time to make the right decisions to ensure success [8]. Owing to the high intensity and speed of the game, only a high level of all coordination abilities allows the player to optimally align the neuromuscular apparatus with the kinaestheticmotor analyser [27].

Reaction is the most frequent focus of analyses in the area of coordination abilities. Owing to the nature of the game, only a high level of reaction to visual and auditory stimuli enables the achievement of championlevel performance. The analysis of literature leads to the conclusion that badminton players present better reaction times compared with control groups [28-31]. Interesting research was also done into the differences in reaction times between men and women of national teams of Turkey, Azerbaijan, Bulgaria, Macedonia, Serbia, and Georgia [24]. In men and women, statistically significant differences between individual countries were demonstrated in the level of visual reaction time for the dominant and non-dominant limb. Furthermore, statistically significant differences in auditory reaction times were documented for the dominant limb in men and the non-dominant limb in women. The study also showed that the reaction times of players from highly ranked countries were better. Therefore, reaction times to auditory and visual stimuli play an important role in the level of achievement in badminton. These findings are partially consistent with our results. High Spearman's rank correlation coefficients were obtained only between the sports skill level and reaction time to visual stimuli (at the limit of statistical significance). Definitely lower correlation coefficients were obtained for auditory and complex reaction times. Sports skill level was most strongly determined by spatial orientation (ca. 42\%). In the regression model, this ability was most important, with its essence being a quick reaction to a moving object and rapid visual reaction time.

An interesting study was conducted by Yuan et al. [32], who determined the importance of the level of visual and motor coordination and reaction time in badminton players. They observed badminton players, gymnasts, and university students from New Zealand. Their findings clearly indicated a higher level of the analysed coordination abilities in badminton players 
compared with the control groups. In our study, we also observed high correlation coefficients between the sports skill level and visual-motor coordination.

Another noteworthy study was performed by Poliszczuk and Mosakowska [25]. The aim of the study was to assess the ability to perceive and process visual information and to anticipate movements in time, as well as to determine the mutual correlations between these characteristics in 9 players of the Polish national badminton team. Two Vienna Test System tests were used in this study: the time-movement anticipation (ZBA) and the peripheral perception test (PP). The authors stated that the dominance of the right upper limb was accompanied by a greater angle of view of the left eye. Despite the dominance of the left eye, a shorter reaction time and more accurate decision-making were observed when exposed to stimuli from the right side.

Another analysis based on a scientific experiment established the relationships between the reaction abilities of badminton players and their perceptual performance in activities related to motor activity $[33,34]$. There was a significant correlation between very good reaction times of the upper and lower limbs. The analyses confirmed the high importance of perception and the effectiveness of motor training in the development of playing skills. The studies demonstrated improved abilities among elite players in the speed and accuracy of movements.

Our findings confirm the importance of coordination abilities in the development of the sports skill level in badminton players, $17-42 \%$ of which is determined by neurofunctional variables.

\section{Conclusions}

The results presented in the study lead to the following conclusions:

1. The results of multidimensional statistical analysis (multiple regression) showed that among the selected abilities with a neurofunctional background, spatial orientation, frequency of movements, and kinaesthetic differentiation had a decisive effect on sports skill level.

2. Sports skill level in badminton players was most strongly determined by spatial orientation (ca. $42 \%$ ), followed by frequency of movements (28\%) and kinaesthetic differentiation (ca. 17\%).

3. The identified coordination abilities seem to be an essential component of the technical and tactical preparation of badminton players. They are directly related to their level of achievement in the sport.

\section{Acknowledgements}

This study is part of the research project 'Level of selected morphofunctional indices in badminton players from the Polish national team and untrained men,' which was supported by statutory funds from the University of Physical Education in Krakow, Institute of Sport Sciences (No. 106/BS/IS/2016).

\section{Disclosure statement}

No author has any financial interest or received any financial benefit from this research.

\section{Conflict of interest}

The authors state no conflict of interest.

\section{References}

1. Phomsoupha M, Laffaye G. The science of badminton: game characteristics, anthropometry, physiology, visual fitness and biomechanics. Sports Med. 2015;45(4): 473-495; doi: 10.1007/s40279-014- 0287-2.

2. Lees A. Science and the major racket sports: a review. J Sports Sci. 2003;21(9):707-732; doi:10.1080/026404 1031000140275.

3. Li S, Zhang Z, Wan B, Wilde B, Shan G. The relevance of body positioning and its training effect on badminton smash. J Sports Sci. 2017:35(4):310-316; doi: 10.1080/02640414.2016.1164332.

4. Bompa TO. Periodization: theory and methodology of training, $4^{\text {th }}$ ed. Champaign: Human Kinetics; 1999.

5. Kuntze G, Mansfield N, Sellers W. A biomechanical analysis of common lunge tasks in badminton. J Sports Sci. 2010;28(2):183-191; doi: 10.1080/026404109034 28533.

6. Sung N-J, Choi J-W, Kim C-H, Lee A, Hong M. Implementation of badminton motion analysis and training system based on IoT sensors. J Internet Comput Serv. 2017;18(4):19-25; doi: 10.7472/jksii.2017.18.4.19.

7. Nadzalan AM, Mohamad NI, Lee JLF, Chinnasee C. Relationship between muscle architecture and badminton-specific physical abilities. Hum Mov. 2018;19(1): 44-50; doi: 10.5114/hm.2018.73611.

8. Chansrisukot G, Suwanthada S, Intiraporn C. Cognitive psychological training in combination with explosive power training can significantly enhance responsiveness of badminton players. J Exerc Physiol Online. 2015;18(4):17-32.

9. Abián-Vicén J, Castanedo A, Abián P, Sampedro J. Temporal and notational comparison of badminton matches between men's singles and women's singles. Int J Perf Anal Spor. 2013;13(2):310-320; doi: 10.1080/ 24748668.2013.11868650.

10. Abián P, Castanedo A, Feng XQ, Sampedro J, AbiánVicén J. Notational comparison of men's singles badminton matches between Olympic Games in Beijing and London. Int J Perf Anal Spor. 2014;14(1):42-53; doi: 10.1080/24748668.2014.11868701. 
11. Laffaye G, Phomsoupha M, Dor F. Changes in the game characteristics of a badminton match: a longitudinal study through the Olympic game finals analysis in men’s singles. J Sports Sci Med. 2015;14(3):584-590.

12. Savarirajan R. Result of heart rate, playing time and performance of Tamilnadu badminton senior ranking players. Int J Sports Sci Fitness. 2016;6(1):43-56.

13. Valldecabres R, Benito A-M, Casal CA, Pablos C. 2015 Badminton World Championship: singles final men's vs women's behaviours. J Hum Sport Exerc. 2017;12(3 proc.):S775-S788; doi: 10.14198/jhse.2017.12.Proc3.01.

14. Faude O, Meyer T, Rosenberger F, Fries M, Huber G, Kindermann W. Physiological characteristics of badminton match play. Eur J Appl Physiol. 2007;100(4): 479-485; doi: 10.1007/s00421-007-0441-8.

15. Ghosh AK. Heart rate and blood lactate responses during execution of some specific strokes in badminton drills. Int J Appl Sports Sci. 2008;20(2):27-36.

16. Ooi CH, Tan A, Ahmad A, Kwong KW, Sompong R, Ghazali KAM, et al. Physiological characteristics of elite and sub-elite badminton players. J Sports Sci. 2009;27(14): 1591-1599; doi: 10.1080/02640410903352907.

17. Tomaszewski P, Kęska A, Tkaczyk J, Nowicki D, Sienkiewicz-Dianzenza E. Somatic characteristics and motor fitness of elite and sub-elite Polish male badminton players. J Sports Med Phys Fitness. 2018;58(10):14561464; doi: 10.23736/S0022-4707.17.07279-6.

18. Rampichini S, Limonta E, Pugliese L, Cè E, Bisconti AV, Gianfelici A, et al. Heart rate and pulmonary oxygen uptake response in professional badminton players: comparison between on-court game simulation and laboratory exercise testing. Eur J Appl Physiol. 2018:118(11): 2339-2347; doi: 10.1007/s00421-018-3960-6.

19. http://www.pzbad.pl/pl/rozgrywki/elita/listy-klasyfikacyjne.html. Accessed: March 2019.

20. Hirtz P, Forschungszirkel “N.A. Bernstein”. Phenomena of human motor development [in German]. Schorndorf: Hofmann; 2007.

21. Sterkowicz S, Jaworski J. Participation in computer games vs. coordination motor abilities and body composition in boys from rural areas of Poland. Hum Mov. 2013;14(1):4-10; doi: 10.2478/v10038-012-0048-8.

22. Ryguła I. The research process in sport sciences [in Polish]. Katowice: AWF; 2014.

23. Van de Water T, Huijgen B, Faber I, Elferink-Gemser M. Assessing cognitive performance in badminton players: a reproducibility and validity study. J Hum Kinet. 2017;55(1):149-159; doi: 10.1515/hukin-2017-0014.

24. Yüksel MF, Tunç GT. Examining the reaction times of international level badminton players under 15 . Sports. 2018;6(1):20; doi: 10.3390/sports6010020.

25. Poliszczuk T, Mosakowska M. Interreactions of peripheral perception and ability of time-movement anticipation in high class competitive badminton players. Stud Phys Cult Tour. 2009;16(3):259-265.

26. http://www.badminton.opole.pl/attachments/article/132/Strategia_badmintona_2020.pdf. Accessed: March 2019.
27. Hülsdünker T, Strüder HK, Mierau A. Visual but not motor processes predict simple visuomotor reaction time of badminton players. Eur J Sport Sci. 2018;18(2): 190-200; doi: 10.1080/17461391.2017.1395912.

28. Bańkosz Z, Nawara H, Ociepa M. Assessment of simple reaction time in badminton players. Trends Sport Sci. 2013;1(20):54-61.

29. Bhabhor M, Vidja K, Dubal G, Padalia M, Jani H, Joshi V. A comparative study of visual reaction time in badminton players and healthy controls. Indian J Appl Basic Med Sci. 2013;15a(20):76-82.

30. Dube SP, Mungal SU, Kulkarni MB. Simple visual reaction time in badminton players: a comparative study. Natl J Physiol Pharm Pharmacol. 2015;5(1):18-20; doi: 10.5455/njppp.2015.5.080720141.

31. Kaplan DS, Akcan F, Çakır Z, Kılıç T, Yıldırım C. Visuomotor and audiomotor reaction time in elite and nonelite badminton players. Eur J Phys Educ Sport Sci. 2017;3(1):84-93; doi: 10.5281/zenodo.293183.

32. Yuan YWY, Fan X, Chin M-K, So RCH. Hand-eye coordination and visual reaction time in elite badminton players and gymnasts. NZ J Sports Med. 1995;23(3): 19-22.

33. Wang S-M, Yan C, Zhang J-C. A research on evaluation target of reaction ability correctly for badminton players in the process of appraising perceptual-motor skill. J Beijing Sport Univ. 2008;31(6):779-781.

34. Wang S-M, Zhang J-C, Yin X-J. A research on performance of perceptual-motor skill training for badminton players. J Beijing Sport Univ. 2009;32(9):46. 